\title{
ON THE MONODROMY GROUP OF EVERYWHERE TANGENT LINES TO THE OCTIC SURFACE IN $\mathbf{P}^{3}$
}

HARRY D'SOUZA

(Communicated by Jonathan M. Rosenberg)

\begin{abstract}
Let $S_{0}$ be an octic surface in $\mathrm{P}^{3}, G=G(1,3)=$ Grassmannian of lines in $\mathbf{P}^{3}$, and $\mathbf{J}=\left\{(x, l) \mid x \in l \cap S_{0}\right\} \subset S_{0} \times G$. Then $\operatorname{dim} \mathbf{J}=5$. Let $\mathbf{L}=\left\{l \mid l \text { is everywhere tangent to } S_{0}\right\}^{-} \subset G$. Let $\pi_{2}: S_{0} \times G \rightarrow G$ be the projection onto the second factor. We denote its restriction to $\mathbf{J}$ also by $\pi_{2}$. Then the locus of everywhere tangent lines is $\pi_{2}(\mathbf{L})$. In this article we show that the monodromy group of these lines is the full symmetric group.
\end{abstract}

Introduction. In this article we show that a general octic surface in $\mathbf{P}^{3}$, has a finite number of everywhere tangent lines and using the Harris technique in $[\mathbf{H}]$, we study the monodromy group of these lines and prove that this group is the full symmetric group.

\section{Notation and background material.}

(0.1) Let $S_{0}$ be an octic surface in $\mathbf{P}^{3}$. First we note that if $S_{0}$ is general then it cannot contain lines. Let $G=G(1,3)=$ Grassmannian of lines in $\mathbf{P}^{3}$. Let $\mathbf{J}=\left\{(x, l) \mid x \in l \cap S_{0}\right\} \subset S_{0} \times G$. We also note that $\operatorname{dim} \mathbf{J}=2+4-1=5$.

(0.2) Definition. A line $l$ in $\mathbf{P}^{3}$ is said to be everywhere tangent to an octic surface $S$ in $\mathbf{P}^{3}$, if the multiplicity at each of the points of intersection of $S$ and $l$ in $\mathbf{P}^{3}$ is at least two.

(0.3) Let $\mathbf{L}=\left\{(x, l) \mid l \text { is everywhere tangent to } S_{0}\right\}^{-} \subset \mathbf{J}$; also let $\pi_{2}: S_{0} \times G \rightarrow G$ be the projection onto the second factor. We denote its restriction to $\mathbf{J}$ also by $\pi_{2}$, then the locus of everywhere tangent lines to $S_{0}$ is $\pi_{2}(\mathrm{~L})$. From now on we denote $\pi_{2}(\mathbf{L})$ by $\mathbf{F}$.

(0.4) Let $X$ be a smooth complex projective variety of dimension $n$.

Let $\mathrm{Hilb}_{c}^{r} X$ denote the curvilinear Hilbert scheme of $r$ points of $X$. Then Hilb $_{c}^{r} X$ is smooth of dimension $n r$ (see [LB] for details).

If $Y$ is a smooth hypersurface in $X$, then there is a natural inclusion $\operatorname{Hilb}_{c}^{r} Y \rightarrow$ Hilb $_{c}^{r} X$ of dimension $(n-1) r$.

Let $\operatorname{Hilb}_{c}^{r} \underline{S} / \mathbf{P}^{N}$ denote the relative (curvilinear) Hilbert scheme of the universal family of octic surfaces parametrized by $\mathbf{P}^{N}$; we note that $N=164$.

The linear system of all octics in $\mathbf{P}^{3}$ is denoted by $W_{8}$, and is of dimension $N=164$. If $S$ denotes a general octic surface in $\mathbf{P}^{3}$ (see $(0.6)$ ), then the relative (curvilinear) Hilbert scheme above is of dimension $2 r+N$.

(0.5) Let $\mathbf{a}=\left(a_{1}, \ldots, a_{k}\right)$ where $a_{i} \leq a_{j}$ where $i<j$ and $a_{i} \geq 2$, and $r=\sum a_{i}=$ 8. We note that $\mathbf{a}=(2,2,2,2) ;(2,2,4) ;(2,3,3) ;(2,6) ;(3,5) ;(4,4) ;(8)$, are the only possibilities.

Received by the editors February 25, 1988.

1980 Mathematics Subject Classification (1985 Revision). Primary 14J25, 14E20, 14N10, 51N35. 
In what follows, the notation is borrowed from [Co], where one can check for details.

Let $\mathbf{a}=\left(b_{1}, \ldots, b_{1}, b_{2}, \ldots, b_{2}, \ldots, b_{p}, \ldots, b_{p}\right)$ where $b_{i}<b_{j}$ for $i<j$, and $k=$ $\sum q_{i}$ where $q_{i}$ denotes the number of $b_{i}$, for $1 \leq i \leq p$. Consider $j_{\mathbf{a}}$ : $\operatorname{Hilb}^{q_{1}} \mathbf{P}^{1} \times$ $\cdots \times$ Hilb $^{q_{p}} \mathbf{P}^{1} \rightarrow \mathrm{Hilb}^{r} \mathbf{P}^{1} \cong \mathbf{P}^{r}$ given by

$$
\left(I_{1}, \ldots, I_{p}\right) \mapsto I_{1}^{b_{1}}, \ldots, I_{p}^{b_{p}}
$$

where $I_{j}$ is the ideal of the zero-dimensional length $q_{j}$ subscheme of $\mathbf{P}^{1}$. We note that $j_{\mathbf{a}}$ is an embedding. Using $j_{\mathbf{a}}$ we can construct a flat family over $G$, denoted by $D_{\mathbf{a}}$, whose fibres are all isomorphic to the image of $j_{\mathbf{a}}$, and $D_{\mathbf{a}}$ embeds naturally in the curvilinear Hilbert scheme $\operatorname{Hilb}_{c}^{r} \mathbf{P}^{3}$, and $\operatorname{dim} D_{\mathbf{a}}=k+4$.

(0.6) Definition. Consider $P_{\mathbf{a}}=D_{\mathbf{a}} \cap \operatorname{Hilb}_{c}^{r} S$, where $S$ is an octic surface in $\mathbf{P}^{3}$. $S$ is said to be general if $P_{\mathbf{a}}$ is empty or $P_{\mathbf{a}}$ has the expected dimension $(k+4)+2 r-3 r=k-4$ for all possible partitions of $8(=\operatorname{deg} S)$.

\section{General results.}

(1.0) Proposition. There exists a nonempty open subset $U$ of $W_{8}\left(\cong \mathbf{P}^{N}\right) N=$ 164 , (see (0.4)) such that any member of $U$ is general in the sense of $(0.6)$.

PROOF. Consider the following commutative diagram

$$
\begin{aligned}
& X \rightarrow \operatorname{Hilb}_{c}^{r} \frac{S}{\downarrow} / \mathbf{P}^{N} \rightarrow \mathbf{P}^{N} \\
& \downarrow \\
& D_{\mathbf{a}} \rightarrow \underset{\downarrow}{\downarrow} \operatorname{Hilb}_{c}^{r} \mathbf{P}^{3} \\
& \downarrow \\
& G
\end{aligned}
$$

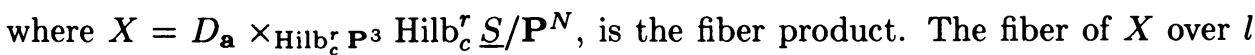
$(\in G)$ consists of all type-a subschemes of $l$ which also lies on some octic surface $S$. Hence the dimension of the fiber is $(N-r)+k=164-8+k=156+k$. Also both $l$ and $S$ lie in some $\mathbf{P}^{3}$. Hence $\operatorname{dim} X=160+k$. Hence the general fiber over $\mathbf{P}^{N}$ is of dimension $160+k-N=k-4$. But the fiber of a general $x \in \mathbf{P}^{N}$ is just $P_{\mathbf{a}}$ (see (0.6)). Hence for an open subset $U$ of $\mathbf{P}^{N}, P_{\mathbf{a}}$ is as desired.

(1.1) CoROllaRY. If $S_{0}$ is a general octic in $\mathbf{P}^{3}$ then $\mathbf{F}$ is a finite set. Moreover only the $(2,2,2,2)$ type (see (0.5)) can possibly occur.

Proof. That $\mathbf{F}$ is finite is immediate from (1.0). Since $S_{0}$ is of degree 8 , we have $l . S_{0}=8$ in $\mathbf{P}^{3}$. Let $\mathbf{a}=\left(a_{1}, \ldots, a_{k}\right)$ where $a_{i} \geq 2$ and $a_{i} \leq a_{j}$ for $i<j$. Let $r=\sum a_{i}=8$, where $a_{i}$ denotes the multiplicity of $l$ at the point of intersection with $S_{0}$, then it is easy to see that the set of $l$ with $\mathbf{a}=(2,2,4) ;(2,3,3) ;(2,6)$; $(3,5) ;(4,4)$ or $(8)$ has $k<4$, (see $(0.5))$ in each case and so for the open set $U$ in (1.0) $P_{\mathrm{a}}$ is empty. In the case $(2,2,2,2) ; k=4$, and so this can occur.

Main results. First we show that $\mathbf{F}$ is not empty, by showing the existence of a general octic surface with an everywhere tangent line. We need the following lemmas and this remark.

(2.0) REMARK. In the following we let $w_{8}=$ the linear system of all octics in $\mathbf{P}^{2}$, and $W_{8}=$ the linear system of all octic surfaces in $\mathbf{P}^{3}$. 
(2.1) LemMA. Given a line $l$ in $\mathbf{P}^{2}$ and 4 distinct points on it, there exists an octic curve, $C$ in $\mathbf{P}^{2}$ such that $m_{p}(l . C)=2$, i.e. the line intersects each of these points with multiplicity 2.

Proof. If $G=G(1,2)=$ Grassmannian of all lines in $\mathbf{P}^{2}$, then $\operatorname{dim} G=2$ and $\operatorname{dim} w_{8}=44$. Let $p_{1}, p_{2}, p_{3}$ and $p_{4}$ denote the 4 specified points on $l$.

Let $w^{\prime}$ be the space of octics having the tangent line $l$ at these 4 points $p_{i}$, then $\operatorname{dim} w^{\prime}=\operatorname{dim} w-\left[2\right.$ for each $\left.p_{i}\right]=44-8=36$. Also the space of all marked lines in $\mathbf{P}^{2}$ passing through 4 marked points $p_{i}$ is of dimension, $\operatorname{dim} \mathbf{P}^{2}+[1$ for each $\left.p_{i}\right]=2+4=6$.

Let $w_{0}$ be the space of octics with everywhere tangent lines in $\mathbf{P}^{2} ;$ then $\operatorname{dim} w_{0}=$ $36+6=42$.

(2.2) LEMMA. Given a line $l$ in $\mathbf{P}^{2}$ and 3 distinct points say $p^{\prime}, p^{\prime \prime}$ and $p^{\prime \prime \prime}$ on it, there exists an octic curve, $C$ in $\mathbf{P}^{2}$ such that $m_{p^{\prime}}(l . C)=2=m_{p^{\prime \prime}}(l . C)$ and $m_{p^{\prime \prime \prime}}(l . C)=4$.

PROOF. Let $w^{\prime}$ be as in (2.1). Then as in (2.1) we see that $\operatorname{dim} w^{\prime}=\operatorname{dim} w-$ [2 for each of $p^{\prime}$ and $p^{\prime \prime}$ and 4 for $\left.p^{\prime \prime \prime}\right]=44-8=36$ and the space of all marked lines in $\mathbf{P}^{2}$ passing through 3 marked points $p^{\prime}, p^{\prime \prime}$ and $p^{\prime \prime \prime}$ is of dimension, $\operatorname{dim} \mathbf{P}^{2}+$ [ 1 for each of $p^{\prime}, p^{\prime \prime}$ and $\left.p^{\prime \prime \prime}\right]=2+3=5$. Thus the dimension of the space of such octics is $36+5=41$.

(2.2.1) Let $w_{1}$ be the space of octics satisfying (2.2) then by (2.2), $\operatorname{dim} w_{1}=41$.

(2.3) LEMMA. For a general octic surface in $\mathbf{P}^{3}$, there are only finitely many $l$ with $\mathbf{a}=(2,2,2,2)$. Moreover the set of such l is nonempty.

ProOF. Consider $\left(\mathbf{P}^{3}\right)^{*}$, and let $\varsigma$ be the tautological bundle on $\left(\mathbf{P}^{3}\right)^{*}$. This bundle is nontrivial. Choose a Zariski open subset, say $A$, of $\left(\mathbf{P}^{3}\right)^{*}$ over which this bundle is trivial.

For $H \in A$, let $w_{S}=\left\{C_{H} \mid C_{H}=H \cap S, S\right.$ a fixed general octic in $\left.\mathbf{P}^{3}\right\} \subset w_{8}$ (see (2.0)), then via the map $\Psi: A \rightarrow w_{S}$ given by $H \mapsto H \cap S$ we see that $\operatorname{dim} w_{S}=3$. Hence if $\mathbf{a}=(2,2,2,2)$, by $(2.1), \operatorname{dim}\left(w_{0} \cap w_{S}\right) \geq \operatorname{dim} w_{0}+\operatorname{dim} w_{S}-\operatorname{dim} w_{8}=$ $42+3-44=1$. Hence $\mathbf{F}$ is (1.0) is a nonempty finite set.

(2.4) LEMMA. Using the notations of (1.1) there exists an octic surface in $\mathbf{P}^{3}$, and a tangent line $l$ with $\mathbf{a}=(0,2,2,4)$.

ProOF. We note that by $(1.1), S_{1}$ cannot be general. Let $\mathbf{a}=(0,2,2,4)$ and $w_{1}$ as in (2.2.1); suppose $C_{1} \in w_{1}$. Let $S_{1}$ be an octic surface in $\mathbf{P}^{3}$ containing $C_{1}$, such a surface $S_{1}$ must exist by (2.2).

(2.5) Let $W_{8}$ be as in (2.0). We note that $\operatorname{dim} W_{8}=164$. Let $S$ be a general octic in $\mathbf{P}^{3}$; let $m_{p}(S . l)$ denote the intersection multiplicity of $S$ and $l$, in $\mathbf{P}^{3}$, and let $I_{8}=\{(S ; p, l) \mid l$ is everywhere tangent to $S$, and $p \in l \cap S\} \subset W_{8} \times \mathbf{P}^{3} \times G$. Let $\pi: I_{8} \rightarrow W_{8}$ and $\eta: I_{8} \rightarrow \mathbf{P}^{3} \times G$ be the projections onto the first and the last two factors respectively.

(2.6). REMARKS. (i) By (1.1), (2.1) and (2.2), $\pi$ is generically finite.

(iii) Since the fibers of $\eta$ are linear spaces of dimension $157, I_{8}$ is irreducible.

(2.7) LEMMA. The monodromy group $M$, of $\pi$ is twice transitive.

ProOF. First we show that $M$ is transitive. Let $U$ be a Zariski open subset of $W_{8}$ over which $\pi$ is unramified, then $V=\pi^{-1}(U)$ is irreducible. Let $S \in U$, 
and let $\left(S ; p^{\prime}, l^{\prime}\right)$ and $\left(S ; p^{\prime \prime}, l^{\prime \prime}\right) \in \pi^{-1}(S)$ with $m_{p^{\prime}}\left(S . l^{\prime}\right)=2$ and $m_{p^{\prime \prime}}\left(S . l^{\prime \prime}\right)=2$, respectively. Draw an arc $\gamma^{\sim}$ on $V$ with $\gamma^{\sim}(0)=\left(S ; p^{\prime}, l^{\prime}\right)$ and $\gamma^{\sim}(1)=\left(S ; p^{\prime \prime}, l^{\prime \prime}\right)$; then the monodromy action associated to the arc $\gamma=\pi \circ \gamma^{\sim}$ will carry $\left(S ; p^{\prime}, l^{\prime}\right)$ to $\left(S ; p^{\prime \prime}, l^{\prime \prime}\right)$. Thus $M$ is transitive.

Now let $S_{0}$ be a general octic with $p_{0} \in S_{0}$, and $\left(p_{0}, l_{0}\right)$ such that $m_{p}\left(S_{0} \cdot l_{0}\right)=$ 2 and $\left(S_{0} ; p_{0}, l_{0}\right) \in I_{8}$, i.e. $l_{0}$ is also everywhere else tangent to $S_{0}$. Let $W_{0}=$ $\pi\left(\eta^{-1}\left(p_{0}, l_{0}\right)\right)=\left\{S \mid m_{p}\left(S . l_{0}\right) \geq 2\right\} \leq W_{8}$; we note that $S_{0} \in W_{0}$ and let $I=$ $\left\{(S ; p, l) \mid m_{p}(S . l) \geq 2, p \neq p_{0}, l \neq l_{0}\right\} \subset I_{8}$, then $\eta$ maps $I$ onto a Zariski open subset $\left\{(p, l) \mid p \neq p_{0}, l \neq l_{0}\right\}$ of $\mathbf{P}^{3} \times G$, and as in (2.6), $I$ is irreducible. Hence every Zariski open subset of $I$ is connected. Suppose $\left(S_{0} ; p^{\prime}, l^{\prime}\right),\left(S_{0} ; p^{\prime \prime}, l^{\prime \prime}\right)$ are two other points lying in $\pi^{-1}\left(S_{0}\right)$, then we can find an arc $\gamma^{\sim}$ in $I$ such that $\gamma^{\sim}(0)=\left(S_{0} ; p^{\prime}, l^{\prime}\right)$ and $\gamma^{\sim}(1)=\left(S_{0} ; p^{\prime \prime}, l^{\prime \prime}\right)$. Note that $\pi^{-1}\left(S_{0}\right)$ has all points except $\left(S_{0} ; p_{0}, l_{0}\right) \in I$; then the monodromy action associated to the arc $\gamma=\pi \circ \gamma^{\sim}$ carries $\left(S_{0} ; p^{\prime}, l^{\prime}\right)$ to $\left(S_{0} ; p^{\prime \prime}, l^{\prime \prime}\right)$ and leaves $\left(S_{0} ; p_{0}, l_{0}\right)$ fixed. Hence the stabilizer of $\left(S_{0} ; p_{0}, l_{0}\right)$ in $M$ acts transitively on the remaining points. Hence $M$ is twice transitive.

(2.8) LEMMA. Let $M$ be as in (2.7), then $M$ contains a simple transposition.

PROOF. By (2.4) we know that there exists an octic surface $S_{1}$ in $W_{8}$, with a line $l$, such that $m_{p^{\prime}}\left(S_{1} . l\right)=2=m_{p^{\prime \prime}}\left(S_{1} . l\right)$ and $m_{p^{\prime \prime \prime}}\left(S_{1} . l\right)=4$. Let $U$ be a Zariski open subset of $W_{8}$, over which $\pi$ is unramified. Let $V=\pi^{-1}(U)$, and let $\Delta$ be an open neighbourhood of $S_{1}$ in $W_{8}$, so chosen, so that the points $\left(S_{1} ; p^{\prime}, l\right),\left(S_{1} ; p^{\prime \prime}, l\right)$ and $\left(S_{1} ; p^{\prime \prime \prime}, l\right)$ have disjoint neighbourhoods $\Delta^{\prime}, \Delta^{\prime \prime}$ and $\Delta^{\prime \prime \prime}$ respectively, each mapping onto $\Delta$, via $\pi$. Since $\Delta^{\prime \prime \prime}$ is irreducible, $\Delta^{\prime \prime \prime} \cap V$ is connected. Since $I_{8}$ is irreducible, it is locally irreducible. Thinking of $\left(S_{1} ; p^{\prime \prime \prime}, l\right)$ as two infinitely near points $q$ and $r$ each with multiplicity two i.e. $m_{q}\left(S_{1}, l\right)=2=m_{r}\left(S_{1}, l\right)$, we can find $\gamma^{\sim}$ in $\Delta^{\prime \prime \prime} \cap V$ such that $\gamma^{\sim}(0)=\left(S_{1} ; q, l\right)$ and $\gamma^{\sim}(1)=\left(S_{1} ; r, l\right)$; hence $\gamma=\pi \circ \gamma^{\sim}$ will fix $\left(S_{1} ; p^{\prime}, l\right)$ and $\left(S_{1} ; p^{\prime \prime}, l\right)$ and $\gamma$ interchanges $q$ and $r$. Hence $M$ contains a simple transposition.

(2.9) THEOREM. The monodromy group $M$, of everywhere tangent lines to a general octic surface in $\mathbf{P}^{3}$, is the full symmetric group.

PROOF. By (2.7) and (2.8), for a general octic surface, the monodromy group $M$, is twice transitive and contains a simple transposition; hence it contains all simple transpositions. So $M$ is necessarily the entire symmetric group.

ACKNOWLEDGEMENTS. The author would like to express his deep gratitude to $\mathrm{K}$. Coombes for the many discussions and the many useful comments on earlier drafts. The author is grateful to S. Colley for providing her preprint and clarifying (1.0), and would also like to thank Sandro Verra for suggesting the problem.

\section{REFERENCES}

[Co] S. Colley, Coincidence formulas for line complexes, Comm. Algebra (to appear).

[H] J. Harris, Galois groups of enumerative problems, Duke Math. J. 46 (1979), 685-724.

[LB] P. Le Barz, Quelques calculs dans les variétés d'alignements, Adv. in Math. 64 (1987), 87-117.

Department of Mathematics, University of Michigan, Flint, Michigan 485022186 